\title{
Application of Improved Particle Swarm Optimization Algorithm in Medium and Long Term power Load Combination Forecasting
}

\author{
Shuguo Zhang ${ }^{\mathrm{a}, \star}$, Yang Su
}

Department of Economic Management, North China Electric Power University, Baoding, China

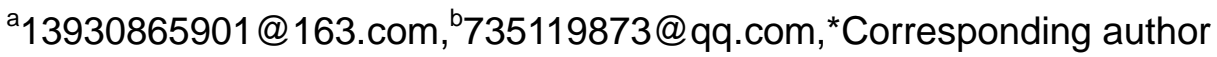

Keywords: Power load forecasting; Combination forecasting; Particle swarm optimization; Inertia weight

\begin{abstract}
There are many methods of power load forecasting, but each method has its own inaccurate influence factors, and the result of the single method is relatively large. Medium and long term power load forecasting influence the development of local planning in the future, so the accuracy of prediction is higher. Based on the two order exponential smoothing, regression analysis and grey prediction model, the comprehensive prediction model based on the three single forecasting methods is built. By using the inertia weight of the particle swarm optimization algorithm to determine the weights, the advantage of the improved combination forecast is obtained by comparison.
\end{abstract}

\section{Introduction}

Power load forecasting is the premise of power system dispatching, safe and stable operation. Accurate power load forecasting can reasonably arrange the operation of the internal engine group of the power grid, and reasonable arrangement of the unit maintenance plans. It is advantageous to improve the scientific planning of regional power grid, and promote the effective utilization of resources to optimize the allocation of regional power energy. At present, the methods of load forecasting are many, and the advantages of each forecasting method are complementary to each other. Through the analysis of the particle motion state, it is concluded that the convergence of the particle swarm can reach the best condition, improve the convergence speed and the global convergence, and reduce the possibility of falling into the local optimum.

\section{Particle Swarm Optimization Algorithm}

Particle swarm optimization (PSO) is a kind of global optimization technology based on biological intelligence, which is inspired by the foraging behavior of birds. The solution space is searched through the mutual sharing information among the particles, and the optimal solution is found. The formula of particle search for PSO algorithm is:

Searched through the mutual sharing information among the particles, and the optimal solution is found. The formula of particle search for PSO algorithm is:

$$
\begin{gathered}
v_{i}^{n+1}=w v_{i}^{n}+c_{1 i} \times r_{1} \times\left(P_{i}^{n}-X_{i}^{n}\right)+c_{2 i} \times r_{2} \times\left(G^{n}-X_{i}^{n}\right) \\
X_{i}^{n+1}=X_{i}^{n}+v_{i}^{n+1}
\end{gathered}
$$

Type: $w_{i s}$ Inertial factor, $r_{1}$ and $r_{2}$ is the random number of random distribution on the interval $(0,1) \cdot c_{1 i}, \quad c_{2 i}$ are learning factors, $x_{i}^{n}$ is the space position of the particle $\mathrm{n}$ in the iteration of $I, v_{i}^{n}$ velocity of particles, $p_{i}^{n}$ is the individual extreme value generated during the search process. $G_{n}$ is the global extreme.

\section{Particle Swarm Optimization Algorithm}

Influence of inertia weight $w$ on particle swarm optimization embodied in the larger value is advantageous to jump out the local optimum and continue to carry on the overall optimization. The 
smaller value is advantageous to the local optimization, and the convergence of the algorithm is accelerated. When the velocity of the particle tends to infinity, the trajectory of the particle is divergent, which leads to the divergence of the trajectories of the whole particle swarm. So the stability of the particle velocity changes has an important influence on the whole particle swarm behavior. The necessary conditions for the study of the stability of the system to be satisfied by the velocity change and the position change of the particle are as follows:

$$
\begin{aligned}
& \left\{\begin{array}{l}
c_{1 i} \times r_{1}+c_{2 i} \times r_{2}>0 \\
1-w \geq 0 \\
2 w+2-c_{1 i} \times r_{1}-c_{2 i} \times r_{2} \geq 0
\end{array}\right. \\
& \left\{\begin{array}{l}
1-w>0 \\
2 w+2-c_{1 i} \times r_{1}-c_{2 i} \times r_{2}>0
\end{array}\right.
\end{aligned}
$$

This paper mainly focuses on the improvement of the inertia weight, An inertia weight particle swarm optimization algorithm based on sine adjustment is proposed. Adjusting coefficient:

$$
w_{t}=0.4+0.5 \sin (\pi t / \text { MaxIterances })
$$

Optimization formula adjusted to

$$
v_{i}^{n+1}=w v_{i}^{n}+[w(t)+1] \times r_{1} \times\left(P_{i}^{n}-X_{i}^{n}\right)+[w(t)+1]\left(2-r_{1}\right) \times r_{2} \times\left(G^{n}-X_{i}^{n}\right)
$$

$r_{1}, r_{2}$ is the random number of random distribution on the interval $(0,1)$, So as to satisfy the formula (1), (2) the stability requirements, so that the algorithm guarantees the convergence performance, optimize.

\section{The Application of the Improved Model in the Power Load Combination Forecasting Model}

The actual value of the first $\mathrm{I}$ in the forecast data is $y_{i}(\mathrm{i}=1,2, \ldots, \mathrm{n}), f_{i \mathrm{k}}$ is the predictive value of the $\mathrm{k}$ method in the first I years, $w_{k}$ weight coefficients for the k method. And $\sum_{i=1}^{m} w_{i}=1, y_{i}^{\prime}$ is combination forecast value, and $y_{i}^{\prime}=\sum_{i=1}^{k} w_{i} f_{i k}$.

The combination forecasting model of fixed weight coefficient is the combination forecasting error square and minimum.

$$
\min E=\sum_{i=1}^{n}\left(y_{i}-\sum_{k=1}^{k} w_{k} f_{i k}\right)^{2}, \quad \text { s.t } \sum_{k=1}^{k} w_{k}=1, \quad w_{k} \geq 0, k=1,2, \ldots, k
$$

Using improved particle swarm optimization algorithm to optimize the combination forecasting model, obtained the combination weight coefficient $w_{i}$, the type (5), can be combined after optimization of the forecast value.

Example Applications. In Guangdong Province, 2006 2015 10 years total electricity consumption for the prediction of basic data, As Table 1 .

Table 1 Annual electricity consumption of 2006 2015 in Guangdong Province Billion kilowatt hours

\begin{tabular}{|c|c|c|c|c|c|}
\hline Particular year & 2006 & 2007 & 2008 & 2009 & 2010 \\
\hline total electricity consumption & 2911 & 3393 & 3505 & 3698 & 4060 \\
\hline Particular year & 2011 & 2012 & 2013 & 2014 & 2015 \\
\hline total electricity consumption & 4399 & 4619 & 4830 & 5235 & 5311 \\
\hline
\end{tabular}

According to the original data of table two, the 1 index smoothing, regression analysis and grey model were established respectively.

Two time exponential smoothing method is used to predict, take $a=0.9$, the predicted value is shown in Table 2. Regression analyse forecasting model with time as controllable variable $x$, Guangdong Province as a whole social demand for electricity as variably. 
$y=268.01 x-534637, R^{2}=0.9901>0.99$, linear features can effectively cover the data, has a very good general.

The GM $(1,1)$ model was established by the whole society of the grey forecasting model, as follows:

$$
\left\{\begin{array}{l}
x^{(1)}(k+1)=6086.27 e^{0.3887 k}-3175.225 \\
x^{(0)}(k+1)=x^{(1)}(k+1)-x^{(1)}(k)
\end{array}\right.
$$

Power load combination forecasting model based on formula (5):

$$
\min E=\sum_{i=1}^{10}\left(y_{i}-\sum_{k=1}^{3} w_{k} f_{i k}\right)^{2}, \quad \text { s.t } \sum_{k=1}^{3} w_{k}=1, \quad w_{k} \geq 0, k=1,2,3
$$

The improved particle algorithm is used to optimize the above formula.

Learning factors $c_{1}$ and $c_{2}$ is 2, a stochastic inertia weight particle swarm optimization algorithm, take $w=0.4+\operatorname{rand}() / 2$, rand() is generated greater than or equal to 0 , less than 1 of the random number, makes the mean value of the iteration process vary between $[0.4,0.9]$, In the search phase, the global optimal particle is 0.9, Other particles are 0.4. Initial population of particles take 100, algorithm runs 145 times to achieve the desired results, The weight of power load combination forecasting model $w_{1}=0.2, w_{2}=0.7, w_{3}=0.1$. The predicted value of the combined forecasting model is obtained as shown in Table 2.

\begin{tabular}{|c|c|c|c|c|c|c|c|c|c|c|}
\hline \multirow{2}{*}{ Model } & \multicolumn{9}{|c|}{ Particular year } & \multirow{2}{*}{$\begin{array}{l}\text { Average relative } \\
\text { error }\end{array}$} \\
\hline & 2007 & 2008 & 2009 & 2010 & 2011 & 2012 & 2013 & 2014 & 2015 & \\
\hline Actual value & 3393 & 3505 & 3698 & 4060 & 4399 & 4619 & 4830 & 5235 & 5310 & - \\
\hline $\begin{array}{l}\text { Exponential } \\
\text { Smoothing }\end{array}$ & 3093 & 3633 & 3645 & 3879 & 4387 & 4737 & 4863 & 5046 & 5602 & $-0.69 \%$ \\
\hline Regression & 3259 & 3527 & 3795 & 4063 & 4331 & 4599 & 4867 & 5135 & 5403 & $-0.22 \%$ \\
\hline gray & 3397 & 3538 & 3703 & 4101 & 4416 & 4701 & 4957 & 5356 & 5539 & $1.52 \%$ \\
\hline Combination & 3239 & 3549 & 3756 & 4030 & 4350 & 4637 & 4875 & 5139 & 5457 & $-0.14 \%$ \\
\hline
\end{tabular}

Table 2 forecast results and average relative error of each model

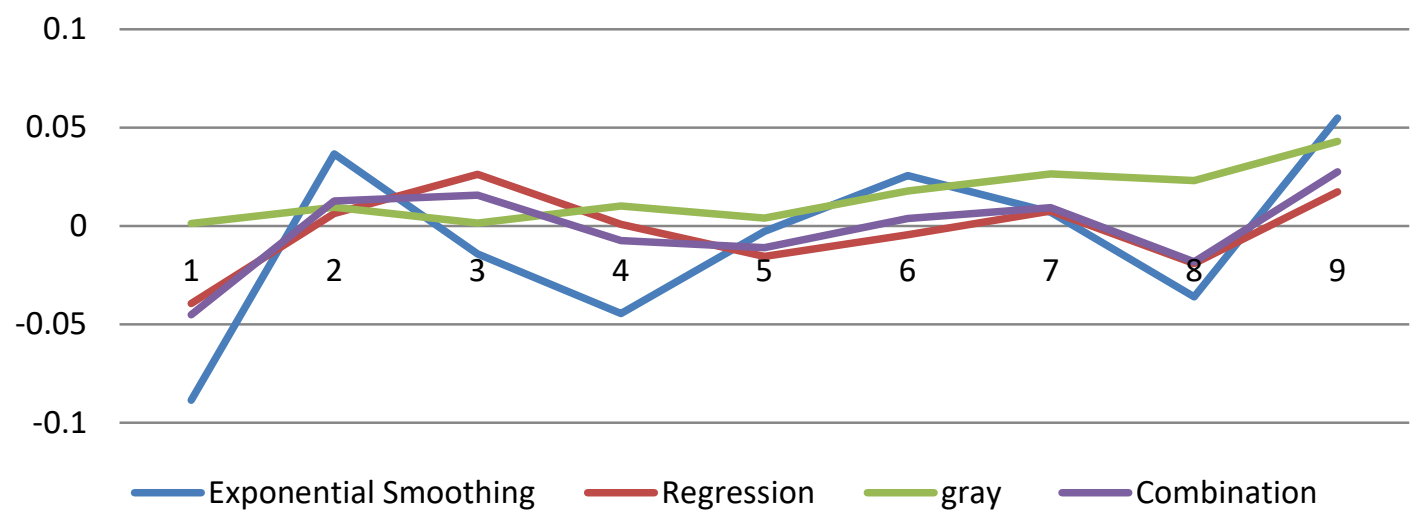

Fig. 1 Comparison of predicted value error of each forecast model

From table 2 and figure 1, the application of improved particle swarm optimization algorithm in the weight of the combined forecasting model, the relative error of the predictive value is less than the exponential smoothing, regression analysis, grey prediction model. That this method converges with high precisionand it is proved that the optimization is feasible. According to the prediction results, the accuracy of the improved particle swarm inertia weight to determine the weight of combined forecasting is higher than that of the single prediction model, and the error is small.

\section{Summary}

Prediction examples show that the improved particle swarm algorithm convergence, global search, and the application in determining the combined power load forecasting model of weights is 
feasible, high prediction accuracy than a single mathematical model, the relative error is small. The improvement in convergence speed and global convergence than the standard PSO was to adjust the inertia value of particle swarm optimization, reduce local optimization, provide the forecast data accuracy, providing an effective prediction method for medium and long term load forecasting.

\section{References:}

[1] Zhao Shuguang, Jiang Changyuan,ShenShigen, Guo. Particle swarm optimization algorithm based on inertia weight sine adjustment[J]. Computer engineering and application, 2012,08:40-42.

[2] Jin Xin, LuoDiansheng, Sun Guangqiang, Zhang Hongxu, Zheng Dun. Model selection and combination method for medium and long term power load forecast[J]. Journal of electric power system and its automation, 2012,04:150-156.

[3] Wu Changyou, WangFulin, Dong Zhigui, Suoruayshya.Improvedparticle swarm optimization algorithm[J]. Power system technology application prediction model in power load combination, 2009,02:27-30.

[4] Bruce Lee, Li Yuxia, Shi Biao, cattle journal, Wang Yu. Application of improved particle swarm optimization algorithm in long term load forecasting [J]. Model in Journal of Wuhan University (Engineering Science Edition), 2011,03:380-382 38

[5] Wu Changyou, Wang Fulin. Improved particle swarm optimization algorithm[J].Rural electrification load forecasting, 2008,08:10-12.

[6] Li Wei, Han Li. Application of combined grey forecasting model in power load forecasting [J]. Journal of Chongqing University (NATURAL SCIENCE EDITION), 2004,01:36-39.

[7] Yu Jianming, Yan Fei, Yang Wenyu, Xia Chao. The Variable Weight Grey Combination Forecasting Model of medium and long term electric power load forecasting [J]. Power system technology, 2005,17:26-29. 